\title{
The avifauna of the Catimbau National Park, an important protected area in the Brazilian semiarid
}

\author{
Flor Maria Guedes Las-Casas ${ }^{1,3,4}$, Iolanda Maria da Silva Pereira' ${ }^{2}$ Lilia D'ark Nunes dos Santos ${ }^{1}$ \\ \& Luciano Nicolás Naka ${ }^{1,3}$
}

\footnotetext{
${ }^{1}$ Departamento de Zoologia, Centro de Biociências, Universidade Federal de Pernambuco (UFPE), Cidade Universitária, Recife, PE, Brazil. Programa de Pós-graduação em Ecologia, Universidade Federal Rural de Pernambuco (UFRPE), Dois Irmãos, Recife, PE, Brazil.

3 Laboratório de Biogeografia, Ecologia e Evolução de Aves, Universidade Federal de Pernambuco, Recife, PE, Brazil.

${ }^{4}$ Corresponding author: flormarialc@hotmail.com
}

Received on 13 August 2018. Accepted on 28 May 2019.

\begin{abstract}
The Catimbau National Park is a protected area, located within the Caatinga Dry Forest, in the central region of the Brazilian state of Pernambuco. This protected area encompasses $-60,000$ ha of an exceptional diversity of habitats, resulting in a high avian diversity, including several rare and endemic species. The park is considered an area of high biological importance and of conservation priority. Despite its relevance for conservation, human degradation due to chronic anthropogenic disturbances (hunting, birds trapping, selective logging, and livestock grazing) has modified the park's natural environments. In 2014, we initiated avian inventories within the park, as part of a long-term ecological research (LTER). Although the avifauna of the park has been described before, our systematic surveys allowed us to have a better understating of the park's avifauna and resulted in several additions to the species list. Here, we update and reevaluate the park's avifauna, discuss the presence of resident and migratory species, and include comments on endemic and rare species that occur within the park's boundaries. We sampled the avifauna through systematic surveys (point counts) and opportunistic observations between 2014 and 2017, including both dry and rainy seasons. We recorded a total of 192 species, including 25 species new to the park's list. During our point counts, we detected 117 species in the dry season, whereas 34 were recorded exclusively during the rainy season. Nearly $10 \%$ of the park's avifauna (19 species) is represented by migratory species, such as Elaenia chilensis and Turdus amaurochalinus. Catimbau National Park is important for the conservation of the Caatinga avifauna, since it harbors endemic, range-restricted, migratory, and globally threatened species. Therefore, we emphasize that environmental education and ecological restoration projects, allied to enforcing environmental laws are urgent for the maintenance of biodiversity and ecosystem services in the Catimbau National Park.
\end{abstract}

KEY-WORDS: Caatinga, long-term ecological research, migratory birds, Neotropical Dry Forests, ornithological inventory.

\section{INTRODUCTION}

The Caatinga Domain (hereafter, Caatinga) represents the largest patch of Seasonally Dry Tropical Forest in the Neotropics (Pennington et al. 2000). Far from representing a single vegetational type, the Caatinga is highly heterogeneous, presenting a wide diversity of ecosystems and habitats. Different combinations of soil, relief, topography and rainfall regimes create a wide variety of habitats (Egler 1951, Sarmiento 1975, Andrade-Lima 1981, Leal et al. 2003). Much of this variation can be found at one particular protected area in the Caatinga: the Catimbau National Park (hereafter, CNP). This exceptional diversity of habitats results in a high diversity of bird species, including several rare and endemic, which is one of the reasons the park is considered an area of high biological importance and of conservation priority (Devenish et al. 2009, Menezes et al. 2012).

Unfortunately, much of the degradation observed within the Caatinga, where over $63 \%$ of its area has already been modified by human activities (Pennington et al. 2009, Araújo \& Silva 2017, Silva \& Barbosa 2017) is also evident at the CNP. The park faces many chronic anthropogenic disturbance pressures as a result of the nearly 300 families that live within the park and depend on livestock grazing and logging to survive (Rito et al. 2017, Arnan et al. 2018). Also, the absence of a welldesigned management plan, mandatory by Brazilian law (SNUC 2002), reflects negatively on the overall conservation of the National Park. At present, CNP presents many degraded areas with different histories of human land use (Cruz et al. 2017, MMA 2018a).

Given the remarkable habitat heterogeneity found at the park, the relatively large topographic variation (500-1100 m), and the rainfall gradient within such a small area (650-1100 mm/yr), Catimbau National Park was selected to establish a Long-term Ecological Research (LTER) Program (http://www.peldcatimbau.org). The 
main purpose of Catimbau's LTER site is to evaluate how chronic anthropogenic disturbances and changes in rainfall regime affect the biota. A total of 20 permanent plots were established, covering most of the topographical, environmental, and anthropogenic disturbance gradient, offering a unique opportunity to understand patterns of diversity in many different biological groups (Rito et al. 2017). Each biological group studied relied on a different sample scheme, depending on the spatial scale desired. To study the avifauna, we established $2 \mathrm{~km}$ transects around each one of the 20 permanent plots, sampled by 10 point counts, systematically established every $200 \mathrm{~m}$.

The avifauna of the CNP is relatively well known due to past surveys (Farias 2009, Sousa et al. 2012). The first ornithologist to present a species list of the park's avifauna, based on non-systematic inventories and opportunistic observations, included 139 species (Farias 2009). A few years later, Sousa et al. (2012) presented a more complete list of the park's avifauna, updating the park's list to 202 species, including important endemic and threatened species, such as Penelope jacucaca and Spinus yarrellii.

In this study, we present the results of three years (2014-2017) of systematic surveys conducted around 20 sites distributed throughout the park, and opportunistic observations conducted elsewhere within the park. We also present a new updated list of the avifauna of the CNP, with relevant information about the avian community, with important records of threatened, migratory, and endemic species. We also provide ecological aspects of species richness and patterns of species composition, highlighting the potential threats found in this protected area and its importance for the conservation of Caatinga birds.

\section{METHODS}

\section{Study area}

The Catimbau National Park $(\sim 60,000$ ha), created by a federal decree on 13 December 2002, is a protected area located within three municipalities (Buíque, Tupanatinga and Ibimirim) in the central region of the Brazilian state of Pernambuco (between $8^{\circ} 24^{\prime} 00^{\prime \prime}$ and $8^{\circ} 36^{\prime} 35^{\prime \prime}$; $37^{\circ} 0^{\prime} 30^{\prime \prime}$ and $37^{\circ} 1^{\prime} 40^{\prime \prime} \mathrm{W}$ ) (Fig. 1). Climate is classified as tropical semiarid, according to Koeppen's classification; showing a mean annual temperature of $23^{\circ} \mathrm{C}$, with a great inter-annually irregularity in rainfall regimes, which vary from 650 to $1100 \mathrm{~mm} /$ year (SNE 2002).

This protected area is located within the Caatinga Domain, a Seasonally Dry Tropical Forest. Most of the park $(70 \%)$ is composed of old-growth vegetation in sandy soils, with five main phytophysiognomies with distinct vegetation structure and floras, including $i$ ) shrubby-arboreal Caatinga generally located on the leeward slopes and at altitudes between 600 and $800 \mathrm{~m}$ a.s.l., ii) shrubby Caatinga with Cerrado elements can be found in many sites of the Chapada São José, both in lower and surrounding areas of the hills and slopes, iii) shrubby Caatinga with elements of rocky fields (campos rupestres) occur in the plateaus and mountain ranges (800 and $1100 \mathrm{~m}$ a.s.l.), iv) evergreen arboreal vegetation (brejos de altitude) at the foothills, and $v$ ) evergreen shrubby Caatinga located on windward slopes between 600 and $800 \mathrm{~m}$ a.s.l. (Rodal et al. 1998, SNE 2002). Systematic and opportunistic methodologies were conducted in these phytophysiognomies, as well as in aquatic environments (lagoons, ponds and temporary pools) found at the CNP (Fig. 2).

\section{Bird survey and analyses}

We conducted avian surveys at the CNP between August 2014 and August 2017, including both the dry and the rainy seasons. We surveyed the avifauna using point counts with unlimited detection radius (Ralph et al. 1996, Bibby et al. 2000, Sutherland et al. 2004). CNP hosts 20 LTER permanent sites (plots), spatially established to remain independent from one another and to account for the climatic and land use variation found at the park (Table 1, Fig. 1). Around each of these 20 sites, we established 2 $\mathrm{km}$-long transects, which we sampled conducting point counts, which were systematically distributed every 200 $\mathrm{m}$, totaling 10 point counts per site and 200 in the park. All localities and point-counts were geo-referenced using a Garmin GPS unit (GPSMAP64). We sampled each point count during $10 \mathrm{~min}$, when all birds detected by sight or sound were recorded. Each site was sampled three times, once during the dry season and twice during the rainy season, totaling 600 point counts. Besides our systematic surveys, we conducted opportunistic observations between point counts and throughout the park's entire area.

Birds were identified by sight and sound by an experienced observer (FMGLC). We used binoculars and digital recorders to observe and document species presence in the area. Taxonomy and nomenclature follow the Brazilian Committee of Ornithological Records (Piacentini et al. 2015). Species were classified according to their conservation and distribution status. Patterns of endemism (Caatinga and northeast Brazil endemics) were based on Pacheco (2004) and Araújo \& Silva (2017). Threatened species were defined according to Brazilian (MMA 2018b) and international red lists (IUCN 2019). Migration status follows Somenzari et al. (2018), who revised migratory patterns for Brazilian birds.

For habitat we used the five types as described by 
Table 1. Permanent plots from the Long-Term Ecological Research (LTER) PELD Catimbau, Brazil.

\begin{tabular}{ccccc}
\hline \multirow{2}{*}{ LTER Sites } & \multicolumn{2}{c}{ Geographic coordinates } & $\begin{array}{c}\text { Annual mean } \\
\text { precipitation }(\mathbf{m m})\end{array}$ & $\begin{array}{c}\text { Altitude } \\
\text { (m a.s.l.) }\end{array}$ \\
\cline { 2 - 3 } Long $(\mathbf{W})$ & -37.1968 & -8.5313 & 647 & 703.0 \\
P02 & -37.3551 & -8.5072 & 591 & 692.2 \\
P04 & -37.3973 & -8.5554 & 516 & 559.8 \\
P07 & -37.2993 & -8.4496 & 578 & 665.9 \\
P08 & -37.2301 & -8.5354 & 647 & 705.4 \\
P10 & -37.2248 & -8.5167 & 673 & 719.8 \\
P11 & -37.3046 & -8.4278 & 540 & 623.3 \\
P14 & -37.3174 & -8.4133 & 510 & 577.8 \\
P15 & -37.3259 & -8.4658 & 555 & 650.6 \\
P16 & -37.2329 & -8.5581 & 940 & 836.8 \\
P17 & -37.3222 & -8.4854 & 653 & 733.3 \\
P20 & -37.2963 & -8.5209 & 843 & 876.2 \\
P21 & -37.3428 & -8.4831 & 552 & 660.8 \\
P22 & -37.3118 & -8.5178 & 785 & 842.1 \\
P23 & -37.238 & -8.4757 & 588 & 655.2 \\
P25 & -37.2346 & -8.4942 & 645 & 698.5 \\
P26 & -37.277 & -8.5113 & 903 & 965.5 \\
P27 & -37.3096 & -8.5372 & 787 & 829.4 \\
P28 & -37.2475 & -8.5708 & 762 & 772.6 \\
P29 & -37.2449 & -8.5166 & 913 & 960.6 \\
P30 & & & &
\end{tabular}

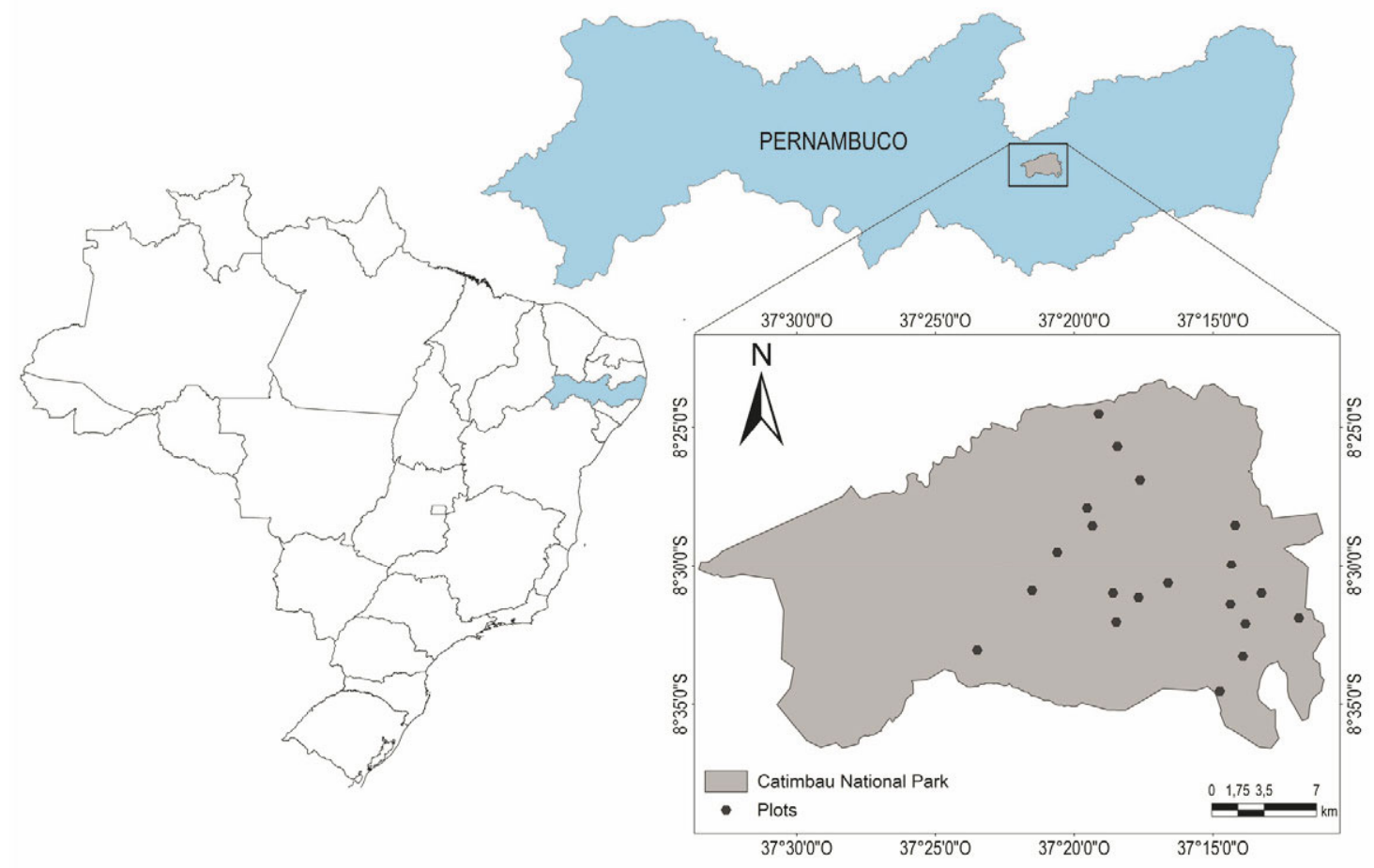

Figure 1. Location of Catimbau National Park, Pernambuco, Brazil. Distribution of the 20 plots used for bird sampling with point counts in PELD Catimbau. 

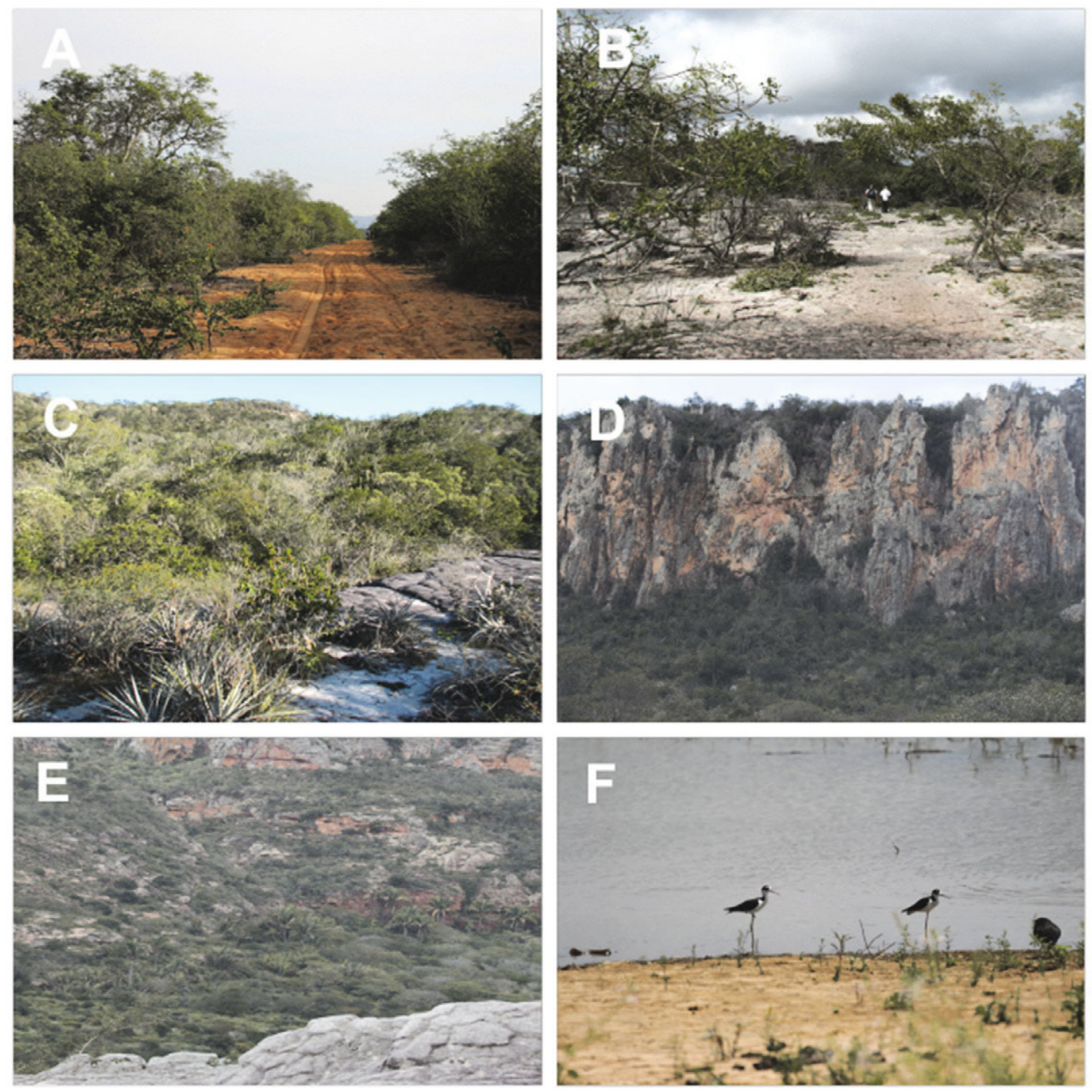

Figure 2. General view of phytophysiognomies and landscapes found at Catimbau National Park, Pernambuco, Brazil. (A) shrubbyarboreal Caatinga; (B) shrubby Caatinga with Cerrado elements; (C) shrubby Caatinga with rocky fields elements (Campos Rupestres); (D) evergreen arboreal vegetation; (E) evergreen shrubby Caatinga; (F) aquatic environment available during the rainy season. Photo author: F.M.G. Las-Casas.

Rodal et al. (1998) and SNE (2002): shrubby-arboreal Caatinga, Caatinga with Cerrado elements, Caatinga with rocky outcrops elements (campos rupestres), evergreen arboreal vegetation and evergreen shrubby Caatinga. The evergreen arboreal vegetation (brejos de altitude) is largely disturbed, especially by agriculture. It presents a typical vegetational composition and can be found in the foothills of the scarpment ( $-800 \mathrm{~m}$ a.s.l.). Habitat categorization for birds was based on our own records, and is applied exclusively for birds detected during this study. Photographs and/or digital recordings were archived at www.wikiaves.com and can be assessed online using the provided voucher numbers (Table 2).

To evaluate sampling effectiveness, we used Chao 1 and Jackknife 1 richness estimators (Magurran 2004), which are based on quantitative data and are known to overcome other estimators in terms of bias and precision
(Gotelli \& Colwell 2010). The total number of species observed at the point counts was represented by a rarefaction curve. These analyses were performed using the software EstimateS 9.1.0 (Colwell 2011). Opportunistic data were excluded from these analyses.

\section{RESULTS}

Our surveys detected 192 species of birds, 25 of which were recorded at the CNP for the first time (Table 2). Point counts resulted in the detection of 155 species ( $-70 \%$ of all species). Opportunistic observations added another 37 species. Bird species detected represented 48 avian families. More than half of the species $(n=106$ or $55.2 \%)$ were passerines, and 86 species $(44.8 \%)$ were non-passerines (Table 2). Sampling around the 20 LTER 
Table 2. List of bird species recorded at the Catimbau National Park, Pernambuco, Brazil. Species recorded by Sousa et al. (2012), and not by us (\#). New records for the park during the present study $\left(^{*}\right)$. Migratory species (MG) and partially migratory (PM). Undefined endemic (End): Caatinga endemic (EC), endemic northeast (EN). Threatened (Thr): "Near Threatened" (NT; IUCN 2019), "Vulnerable" (VU; MMA 2018). Habitat: aquatic environment (AE), shrubby arboreal Caatinga (SA), shrubby Caatinga with Cerrado elements (SC), shrubby Caatinga and rocky fields (RC), evergreen arboreal Caatinga (EA), evergreen shrubby Caatinga (ES).

\begin{tabular}{|c|c|c|c|c|}
\hline Family and species & English names & End/Thr & Habitats & Documentation \\
\hline \multicolumn{5}{|l|}{ TINAMIDAE } \\
\hline Crypturellus noctivagus zabele ${ }^{\#}$ & Yellow-legged Tinamou & EN/VU,NT & & \\
\hline Crypturellus parvirostris & Small-billed Tinamou & & SA/SC/EA/ES & \\
\hline Crypturellus tataupa & Tataupa Tinamou & & SA/SC/EA/ES & \\
\hline Nothura boraquira & White-bellied Nothura & & SA/ES & \\
\hline Nothura maculosa & Spotted Nothura & & SA & \\
\hline Rhynchotus rufescens ${ }^{\#}$ & Red-winged Tinamou & & & \\
\hline \multicolumn{5}{|l|}{ ANATIDAE } \\
\hline Dendrocygna viduata & White-faced Whistling-Duck & & $\mathrm{AE}$ & WA2919491 \\
\hline Cairina moschata & Muscovy Duck & & & \\
\hline Amazonetta brasiliensis ${ }^{*}$ & Brazilian Teal & & & \\
\hline \multicolumn{5}{|l|}{ CRACIDAE } \\
\hline Penelope superciliaris ochromitra* & Rusty-margined Guan & EN & EA/ES & \\
\hline Penelope jacucaca & White-browed Guan & $\mathrm{EC} / \mathrm{VU}$ & & \\
\hline Ortalis araucuan & East Brazilian Chachalaca & & $\mathrm{AE}$ & \\
\hline \multicolumn{5}{|l|}{ PODICIPEDIDAE } \\
\hline Tachybaptus dominicus* & Least Grebe & & $\mathrm{AE}$ & \\
\hline Podilymbus podiceps" & Pied-billed Grebe & & & \\
\hline \multicolumn{5}{|l|}{ PHALACROCORACIDAE } \\
\hline Nannopterum brasilianus ${ }^{\#}$ & Neotropic Cormorant & & & \\
\hline \multicolumn{5}{|l|}{ ARDEIDAE } \\
\hline Tigrisoma lineatum ${ }^{*}$ & Rufescent Tiger-Heron & & & \\
\hline Nycticorax nycticorax" & Black-crowned Night-Heron & & & \\
\hline Butorides striata ${ }^{\#}$ & Striated Heron & & & \\
\hline Bubulcus ibis & Cattle Egret & & SA & \\
\hline Ardea alba & Great Egret & & $\mathrm{AE}$ & \\
\hline Egretta thula & Snowy Egret & & $\mathrm{AE}$ & \\
\hline \multicolumn{5}{|l|}{ CATHARTIDAE } \\
\hline Cathartes aura & Turkey Vulture & & SA/SC/RC/EA/ES & \\
\hline Cathartes burrovianus & Lesser Yellow-headed Vulture & & SA/SC/RC/EA/ES & WA2104773 \\
\hline Coragyps atratus & Black Vulture & & SA/SC/RC/EA/ES & WA2104774 \\
\hline Sarcoramphus papa* & King Vulture & & SA/SC/RC/EA/ES & WA1467218 \\
\hline \multicolumn{5}{|l|}{ ACCIPITRIDAE } \\
\hline Gampsonyx swainsonii & Pearl Kite & & SA/ES & WA1874623 \\
\hline Elanus leucurus & White-tailed Kite & & SA & \\
\hline Ictinia plumbea & Plumbeous Kite & & & \\
\hline Geranospiza caerulescens & Crane Hawk & & SA & \\
\hline
\end{tabular}




\begin{tabular}{|c|c|c|c|c|}
\hline Family and species & English names & End/Thr & Habitats & Documentation \\
\hline Heterospizias meridionalis* & Savanna Hawk & & SA & \\
\hline Urubitinga urubitinga* & Great Black Hawk & & SA & \\
\hline Rupornis magnirostris & Roadside Hawk & & SA/SC/RC/EA/ES & WA2427047 \\
\hline Parabuteo unicinctus* & Harris's Hawk & & SA & WA2560060 \\
\hline Geranoaetus albicaudatus & White-tailed Hawk & & SA & \\
\hline Geranoaetus melanoleucus & Black-chested Buzzard-Eagle & & $\mathrm{SA} / \mathrm{RC}$ & WA2101247 \\
\hline Buteo nitidus" & Gray-lined Hawk & & & \\
\hline Buteo brachyurus & Short-tailed Hawk & & SA/EA & \\
\hline Buteo albonotatus" & Zone-tailed Hawk & & & \\
\hline \multicolumn{5}{|l|}{ RALLIDAE } \\
\hline Aramides mangle $e^{\#}$ & Little Wood-Rail & & & \\
\hline Aramides cajaneus ${ }^{\sharp}$ & Gray-necked Wood-Rail & & & \\
\hline Pardirallus nigricans ${ }^{\#}$ & Blackish Rail & & & \\
\hline Gallinula galeata & Common Gallinule & & $\mathrm{AE}$ & \\
\hline Porphyriops melanops & Spot-flanked Gallinule & & $\mathrm{AE}$ & \\
\hline Porphyrio martinicus $^{\mathrm{PM}}$ & Purple Gallinule & & $\mathrm{AE}$ & WA2951832 \\
\hline \multicolumn{5}{|l|}{ CHARADRIIDAE } \\
\hline Vanellus chilensis & Southern Lapwing & & SA & \\
\hline \multicolumn{5}{|l|}{ RECURVIROSTRIDAE } \\
\hline Himantopus mexicanus* & Black-necked Stilt & & & WA2490975 \\
\hline \multicolumn{5}{|l|}{ JACANIDAE } \\
\hline Jacana jacana & Wattled Jacana & & $\mathrm{AE}$ & \\
\hline \multicolumn{5}{|l|}{ COLUMBIDAE } \\
\hline Columbina minuta & Plain-breasted Ground-Dove & & SA/EA/ES/SC & \\
\hline Columbina talpacoti & Ruddy Ground-Dove & & EA/ES & \\
\hline Columbina squammata & Scaled Dove & & SA/EA/ES/SC & \\
\hline Columbina picui & Picui Ground-Dove & & SA/EA/ES/SC & WA1471673 \\
\hline Claravis pretiosa* & Blue Ground-Dove & & SA & \\
\hline Columba livia* & Rock Pigeon & & & \\
\hline Patagioenas picazuro & Picazuro Pigeon & & SA/EA/ES & \\
\hline Zenaida auriculata & Eared Dove & & SA & WA2723505 \\
\hline Leptotila verreauxi & White-tipped Dove & & SA/EA/ES/SC & \\
\hline Leptotila rufaxilla & Gray-fronted Dove & & EA & \\
\hline \multicolumn{5}{|l|}{ CUCULIDAE } \\
\hline Micrococcyx cinereus ${ }^{* \mathrm{MG}}$ & Ash-colored Cuckoo & & SA & \\
\hline Piaya cayana & Squirrel Cuckoo & & SA/EA/ES/SC & WA2106950 \\
\hline Coccyzus melacoryphus ${ }^{\mathrm{MG}}$ & Dark-billed Cuckoo & & SA/EA/ES/SC & WA2850701 \\
\hline Crotophaga major & Greater Ani & & & \\
\hline Crotophaga ani & Smooth-billed Ani & & SA & \\
\hline Guira guira & Guira Cuckoo & & SA & WA2049012 \\
\hline Tapera naevia & Striped Cuckoo & & SA & \\
\hline \multicolumn{5}{|l|}{ TYTONIDAE } \\
\hline Tyto furcata & American Barn Owl & & SA & \\
\hline
\end{tabular}




\begin{tabular}{|c|c|c|c|c|}
\hline Family and species & English names & End/Thr & Habitats & Documentation \\
\hline \multicolumn{5}{|l|}{ STRIGIDAE } \\
\hline Megascops choliba & Tropical Screech-Owl & & SA & \\
\hline Glaucidium brasilianum & Ferruginous Pygmy-Owl & & SA/EA/ES & WA2677373 \\
\hline Athene cunicularia & Burrowing Owl & & SA & WA2290250 \\
\hline \multicolumn{5}{|l|}{ NYCTIBIIDAE } \\
\hline Nyctibius griseus & Common Potoo & & SA & \\
\hline \multicolumn{5}{|l|}{ CAPRIMULGIDAE } \\
\hline Antrostomus rufus ${ }^{\#}$ & Rufous Nightjar & & & \\
\hline Nyctidromus albicollis & Common Pauraque & & SA & \\
\hline Nyctidromus hirundinaceus & Pygmy Nightjar & EC & SA & \\
\hline Hydropsalis parvula ${ }^{* \mathrm{PM}}$ & Little Nightjar & & SA & WA2723643 \\
\hline Hydropsalis longirostris* & Band-winged Nightjar & & SA & \\
\hline Hydropsalis torquata & Scissor-tailed Nightjar & & SA & \\
\hline Nannochordeiles pusillus novaesi & Least Nighthawk & EN & SA & \\
\hline Chordeiles acutipennis ${ }^{*}$ & Lesser Nighthawk & & & \\
\hline \multicolumn{5}{|l|}{ APODIDADE } \\
\hline Tachornis squamata & Fork-tailed Palm-Swift & & SA/EA & \\
\hline \multicolumn{5}{|l|}{ TROCHILIDAE } \\
\hline Anopetia gounellei & Broad-tipped Hermit & EC & SA/ES & \\
\hline Phaethornis pretrei & Planalto Hermit & & SA/EA/ES & \\
\hline Eupetomena macroura & Swallow-tailed Hummingbird & & SA & WA1989371 \\
\hline Anthracothorax nigricollis" & Black-throated Mango & & & \\
\hline Chrysolampis mosquitus & Ruby-topaz Hummingbird & & SA & WA1874630 \\
\hline Chlorostilbon lucidus & Glittering-bellied Emerald & & SA/SC/RC/EA/ES & WA2918587 \\
\hline Polytmus guainumbi* & White-tailed Goldenthroat & & & \\
\hline Amazilia fimbriata* & Glittering-throated Emerald & & SA & WA2490872 \\
\hline Amazilia lactea* & Sapphire-spangled Emerald & & SA & \\
\hline Heliomaster squamosus & Stripe-breasted Starthroat & & SA/SC & WA2918598 \\
\hline \multicolumn{5}{|l|}{ TROGONIDAE } \\
\hline Trogon curucui & Blue-crowned Trogon & & SA/EA/ES & WA3273333 \\
\hline \multicolumn{5}{|l|}{ ALCEDINIDAE } \\
\hline Chloroceryle americana & Green Kingfisher & & & \\
\hline \multicolumn{5}{|l|}{ BUCCONIDAE } \\
\hline Nystalus maculatus & Spot-backed Puffbird & & SA/SC/RC/EA/ES & WA2346838 \\
\hline \multicolumn{5}{|l|}{ PICIDAE } \\
\hline Picumnus fulvescens & Tawny Piculet & EN/NT & SA/SC/RC/EA/ES & WA2687064 \\
\hline Veniliornis passerinus & Little Woodpecker & & SA/SC/RC/EA/ES & \\
\hline Piculus chrysochloros & Golden-green Woodpecker & & SA & \\
\hline Colaptes melanochloros & Green-barred Woodpecker & & SA/SC/RC/EA/ES & \\
\hline \multicolumn{5}{|l|}{ CARIAMIDAE } \\
\hline Cariama cristata & Red-legged Seriema & & SA/SC & \\
\hline \multicolumn{5}{|l|}{ FALCONIDAE } \\
\hline Caracara plancus & Southern Caracara & & $\mathrm{SA} / \mathrm{SC}$ & \\
\hline Milvago chimachima & Yellow-headed Caracara & & SA/SC & \\
\hline
\end{tabular}




\begin{tabular}{|c|c|c|c|c|}
\hline Family and species & English names & End/Thr & Habitats & Documentation \\
\hline Herpetotheres cachinnans & Laughing Falcon & & SA/SC/EA/ES & WA1635330 \\
\hline Micrastur ruficollis & Barred Forest-Falcon & & SA/SC/EA/ES & \\
\hline Falco sparverius & American Kestrel & & SA/SC/EA/ES & \\
\hline Falco rufigularis & Bat Falcon & & $\mathrm{RC}$ & \\
\hline Falco femoralis & Aplomado Falcon & & SA/SC/EA/ES & \\
\hline Falco peregrinus ${ }^{* \mathrm{MG}}$ & Peregrine Falcon & & SA/ES & \\
\hline \multicolumn{5}{|l|}{ PSITTACIDAE } \\
\hline Primolius maracana ${ }^{*}$ & Blue-winged Macaw & & & \\
\hline $\begin{array}{l}\text { Thectocercus acuticaudatus } \\
\text { haemorrhous }\end{array}$ & Blue-crowned Parakeet & EN & SA/ES & \\
\hline Eupsittula cactorum & Cactus Parakeet & EC & SA/SC/RC/EA/ES & WA2106953 \\
\hline Forpus xanthopterygius & Blue-winged Parrotlet & & SA/SC/EA/ES & WA1989370 \\
\hline Amazona aestiva & Turquoise-fronted Parrot & & SA/EA/ES & \\
\hline \multicolumn{5}{|l|}{ THAMNOPHILIDAE } \\
\hline Myrmorchilus strigilatus strigilatus & Stripe-backed Antbird & EN & SA/SC/RC & \\
\hline Formicivora melanogaster bahiae & Black-bellied Antwren & EN & $\mathrm{SA} / \mathrm{SC} / \mathrm{RC} / \mathrm{EA} / \mathrm{ES}$ & WA1467784 \\
\hline Herpsilochmus sellowi* & Caatinga Antwren & EN & $\mathrm{SA} / \mathrm{RC}$ & WA2113554 \\
\hline Herpsilochmus atricapillus" & Black-capped Antwren & & & \\
\hline Sakesphorus cristatus & Silvery-cheeked Antshrike & $\mathrm{EC}$ & $\mathrm{SA} / \mathrm{SC} / \mathrm{RC}$ & WA2850912 \\
\hline Thamnophilus capistratus & Caatinga Antshrike & $\mathrm{EC}$ & SA/SC/RC & WA2851005 \\
\hline Thamnophilus torquatus & Rufous-winged Antshrike & & SA & \\
\hline Thamnophilus pelzelni & Planalto Slaty-Antshrike & & SA/SC/EA/ES & \\
\hline Taraba major & Great Antshrike & & $\mathrm{SA} / \mathrm{SC} / \mathrm{RC} / \mathrm{ES}$ & \\
\hline \multicolumn{5}{|l|}{ GRALLARIIDAE } \\
\hline Hylopezus ochroleucus & White-browed Antpitta & $\mathrm{EC} / \mathrm{NT}$ & $\mathrm{SA} / \mathrm{SC} / \mathrm{RC} / \mathrm{EA} / \mathrm{ES}$ & WA2308551 \\
\hline \multicolumn{5}{|l|}{ DENDROCOLAPTIDAE } \\
\hline Sittasomus griseicapillus" & Olivaceous Woodcreeper & & & \\
\hline Campylorhamphus trochilirostris & Red-billed Scythebill & & SA & \\
\hline Dendroplex picus* & Straight-billed Woodcreeper & & SA & \\
\hline Lepidocolaptes angustirostris & Narrow-billed Woodcreeper & & $\mathrm{SA} / \mathrm{SC} / \mathrm{RC} / \mathrm{EA} / \mathrm{ES}$ & WA2490963 \\
\hline \multicolumn{5}{|l|}{ FURNARIIDAE } \\
\hline Furnarius figulus & Wing-banded Hornero & & SA & \\
\hline Furnarius leucopus & Pale-legged Hornero & & SA/ES & \\
\hline Pseudoseisura cristata & Caatinga Cacholote & EN & SA & \\
\hline $\begin{array}{l}\text { Phacellodomus rufifrons rufifrons/ } \\
\text { specularis }\end{array}$ & Rufous-fronted Thornbird & EN & SA/SC/EA/ES & \\
\hline Certhiaxis cinnamomeus & Yellow-chinned Spinetail & & $\mathrm{SA} / \mathrm{SC} / \mathrm{RC}$ & \\
\hline Synallaxis hellmayri & Red-shouldered Spinetail & $\mathrm{EC}$ & $\mathrm{SA} / \mathrm{SC} / \mathrm{RC}$ & \\
\hline Synallaxis frontalis & Sooty-fronted Spinetail & & $\mathrm{SA} / \mathrm{SC} / \mathrm{RC}$ & \\
\hline Synallaxis albescens & Pale-breasted Spinetail & & SA & \\
\hline Synallaxis hypospodia $a^{\#}$ & Cinereous-breasted Spinetail & & & \\
\hline Synallaxis scutata ${ }^{*}$ & Ochre-cheeked Spinetail & & & \\
\hline Cranioleuca semicinerea & Gray-headed Spinetail & & SA/EA/ES & \\
\hline
\end{tabular}




\begin{tabular}{|c|c|c|c|c|}
\hline Family and species & English names & End/Thr & Habitats & Documentation \\
\hline \multicolumn{5}{|l|}{ TITYRIDAE } \\
\hline Pachyramphus viridis & Green-backed Becard & & SA/SC & \\
\hline Pachyramphus polychopterus ${ }^{\mathrm{PM}}$ & White-winged Becard & & SA/SC/ES & WA2918602 \\
\hline Pachyramphus validus ${ }^{\sharp \mathrm{PM}}$ & Crested Becard & & & \\
\hline Xenopsaris albinucha & White-naped Xenopsaris & & SA & WA2491020 \\
\hline \multicolumn{5}{|l|}{ RHYNCHOCYCLIDAE } \\
\hline Tolmomyias flaviventris & Yellow-breasted Flycatcher & & SA/SC/EA/ES & \\
\hline Todirostrum cinereum & Common Tody-Flycatcher & & SA/SC/RC/ES & \\
\hline Hemitriccus margaritaceiventer & Pearly-vented Tody-tyrant & & SA/SC/RC & WA2101248 \\
\hline \multicolumn{5}{|l|}{ TYRANNIDAE } \\
\hline Hirundinea ferruginea & Cliff Flycatcher & & SA/RC & WA2288299 \\
\hline Stigmatura napensis bahiae & Lesser Wagtail-Tyrant & EN & SA/SC & WA2678822 \\
\hline Euscarthmus meloryphus & Tawny-crowned Pygmy-Tyrant & & SA/SC/RC & \\
\hline Camptostoma obsoletum & Southern Beardless-Tyrannulet & & SA/SC/RC/EA/ES & WA2347009 \\
\hline Elaenia flavogaster & Yellow-bellied Elaenia & & EA/ES & \\
\hline Elaenia spectabilis $^{\mathrm{PM}}$ & Large Elaenia & & SA/SC/RC/ES & \\
\hline Elaenia chilensis $^{\mathrm{MG}}$ & Chilean Elaenia & & SA/SC/RC/ES & WA2677969 \\
\hline Suiriri suiriri bahiae* & Suiriri Flycatcher & EN & SA & WA1874629 \\
\hline Myiopagis viridicata ${ }^{\mathrm{PM}}$ & Greenish Elaenia & & SA/SC/ES & \\
\hline Phaeomyias murina ${ }^{\mathrm{ND}}$ & Mouse-colored Tyrannulet & & SA/SC/RC/ES & WA2850711 \\
\hline Phyllomyias fasciatus cearae & Planalto Tyrannulet & EN & SA/SC/ES & \\
\hline Serpophaga subcristata* & White-crested Tyrannulet & & SA/ES & WA2678868 \\
\hline Myiarchus swainsoni ${ }^{* P M}$ & Swainson's Flycatcher & & SA & \\
\hline Myiarchus ferox & Short-crested Flycatcher & & EA/ES & \\
\hline Myiarchus tyrannulus & Brown-crested Flycatcher & & SA/SC/RC/EA/ES & WA1989368 \\
\hline Casiornis fuscus $^{\mathrm{PM}}$ & Ash-throated Casiornis & & SA/ES & WA2490796 \\
\hline Pitangus sulphuratus & Great Kiskadee & & SA/SC/ES & \\
\hline Machetornis rixosa & Cattle Tyrant & & SA/ES & \\
\hline Myiodynastes maculatus ${ }^{\mathrm{PM}}$ & Streaked Flycatcher & & SA/ES & WA2490928 \\
\hline Megarynchus pitangua & Boat-billed Flycatcher & & SC/EA/ES & \\
\hline Myiozetetes similis & Social Flycatcher & & SA/SC/EA & \\
\hline Tyrannus melancholicus & Tropical Kingbird & & SA/SC/RC/EA/ES & \\
\hline Tyrannus savana*PM & Fork-tailed Flycatcher & & SA & \\
\hline Empidonomus varius ${ }^{\mathrm{M} M}$ & Variegated Flycatcher & & SA/SC/RC/EA/ES & WA2851003 \\
\hline Myiophobus fasciatus $^{\mathrm{MM}}$ & colored Flycatcher & & SA/SC/RC/ES & \\
\hline Sublegatus modestus ${ }^{\mathrm{PM}}$ & Southern Scrub-Flycatcher & & SA/ES & \\
\hline Fluvicola albiventer & Black-backed Water-Tyrant & & $\mathrm{AE}$ & WA2918597 \\
\hline Fluvicola nengeta & Masked Water-Tyrant & & $\mathrm{SA} / \mathrm{ES}$ & \\
\hline Arundinicola leucocephala & White-headed Marsh Tyrant & & $\mathrm{AE}$ & WA2288334 \\
\hline Cnemotriccus fuscatus & Fuscous Flycatcher & & SA/SC/EA/ES & WA1635342 \\
\hline Knipolegus nigerrimus hoflingi & Velvety Black-Tyrant & EN & $\mathrm{SA} / \mathrm{RC}$ & WA2918592 \\
\hline Xolmis irupero niveus & White Monjita & EN & SA & \\
\hline
\end{tabular}




\begin{tabular}{|c|c|c|c|c|}
\hline Family and species & English names & End/Thr & Habitats & Documentation \\
\hline \multicolumn{5}{|l|}{ VIREONIDAE } \\
\hline Cyclarhis gujanensis & Rufous-browed Peppershrike & & SA/SC/RC/ES & \\
\hline Hylophilus amaurocephalus & Gray-eyed Greenlet & & SA/SC/RC/ES & \\
\hline Vireo chivi ${ }^{\mathrm{PM}}$ & Chivi Vireo & & SA/SC/RC/ES & \\
\hline \multicolumn{5}{|l|}{ CORVIDAE } \\
\hline Cyanocorax cyanopogon & White-naped Jay & & SA/SC/RC/ES & \\
\hline \multicolumn{5}{|l|}{ HIRUNDINIDAE } \\
\hline Stelgidopteryx ruficollis ${ }^{\sharp \mathrm{PM}}$ & $\begin{array}{l}\text { Southern Rough-winged } \\
\text { Swallow }\end{array}$ & & & \\
\hline Progne chalybea ${ }^{\mathrm{PM}}$ & Gray-breasted Martin & & SA & \\
\hline \multicolumn{5}{|l|}{ TROGLODYTIDAE } \\
\hline Troglodytes musculus & Southern House Wren & & $\mathrm{SA} / \mathrm{SC} / \mathrm{RC} / \mathrm{EA} / \mathrm{ES}$ & WA2683268 \\
\hline Pheugopedius genibarbis & Moustached Wren & & & \\
\hline Cantorchilus longirostris bahiae & Long-billed Wren & EN & $\mathrm{SA} / \mathrm{SC} / \mathrm{RC}$ & \\
\hline \multicolumn{5}{|l|}{ POLIOPTILIDAE } \\
\hline Polioptila plumbea & Tropical Gnatcatcher & & $\mathrm{SA} / \mathrm{SC} / \mathrm{RC} / \mathrm{EA} / \mathrm{ES}$ & WA2101250 \\
\hline \multicolumn{5}{|l|}{ TURDIDAE } \\
\hline Turdus leucomelas & Pale-breasted Thrush & & $\mathrm{EA} / \mathrm{ES}$ & \\
\hline Turdus rufiventris & Rufous-bellied Thrush & & SA/SC/RC/EA/ES & \\
\hline Turdus amaurochalinus ${ }^{\mathrm{PM}}$ & Creamy-bellied Thrush & & SA/SC & \\
\hline \multicolumn{5}{|l|}{ MIMIDAE } \\
\hline Mimus saturninus arenaceus & Chalk-browed Mockingbird & EN & SA/SC/RC/ES & WA2723585 \\
\hline \multicolumn{5}{|l|}{ MOTACILLIDAE } \\
\hline Anthus lutescens & Yellowish Pipit & & ES & \\
\hline \multicolumn{5}{|l|}{ PASSERELLIDAE } \\
\hline Zonotrichia capensis & Rufous-collared Sparrow & & SA/SC/RC & WA1467779 \\
\hline Ammodramus humeralis & Grassland Sparrow & & SA & \\
\hline \multicolumn{5}{|l|}{ PARULIDAE } \\
\hline Setophaga pitiayumi & Tropical Parula & & SA/ES & \\
\hline Myiothlypis flaveola & Flavescent Warbler & & SA/EA/ES & \\
\hline \multicolumn{5}{|l|}{ ICTERIDAE } \\
\hline Icterus pyrrhopterus & Variable Oriole & & SA/SC/RC & WA2918615 \\
\hline Icterus jamacaii & Campo Troupial & EN & SA/SC/RC & WA2106592 \\
\hline Chrysomus ruficapillus & Chestnut-capped Blackbird & & SA & \\
\hline Agelaioides fringillarius & Pale Baywing & EN & SA & \\
\hline Molothrus bonariensis & Shiny Cowbird & & SA & \\
\hline Sturnella superciliaris & White-browed Meadowlark & & SA & \\
\hline \multicolumn{5}{|l|}{ THRAUPIDAE } \\
\hline Schistochlamys ruficapillus* & Cinnamon Tanager & & $\mathrm{RC}$ & WA2113557 \\
\hline Paroaria dominicana & Red-cowled Cardinal & EC & SA/SC & WA2918606 \\
\hline Tangara sayaca & Sayaca Tanager & & $\mathrm{SA} / \mathrm{SC} / \mathrm{RC} / \mathrm{EA} / \mathrm{ES}$ & \\
\hline Tangara palmarum & Palm Tanager & & $\mathrm{EA} / \mathrm{ES}$ & \\
\hline Tangara cayana & Burnished-buff Tanager & & SA/SC/RC/EA/ES & \\
\hline Nemosia pileata & Hooded Tanager & & SA/SC/RC/EA/ES & \\
\hline
\end{tabular}




\begin{tabular}{|c|c|c|c|c|}
\hline Family and species & English names & End/Thr & Habitats & Documentation \\
\hline Compsothraupis loricata & Scarlet-throated Tanager & EN & $\mathrm{SA} / \mathrm{SC} / \mathrm{RC}$ & WA1635323 \\
\hline Conirostrum speciosum & Chestnut-vented Conebill & & SC/EA/ES & \\
\hline Sicalis flaveola & Saffron Finch & & SA & \\
\hline Sicalis luteola & Grassland Yellow-Finch & & SA & \\
\hline Volatinia jacarina & Blue-black Grassquit & & $\mathrm{SA} / \mathrm{RC}$ & WA2105977 \\
\hline Coryphospingus pileatus & Pileated Finch & & $\mathrm{SA} / \mathrm{SC} / \mathrm{RC}$ & WA2850094 \\
\hline Tachyphonus rufus & White-lined Tanager & & $\mathrm{SA} / \mathrm{SC} / \mathrm{RC} / \mathrm{ES}$ & WA2105976 \\
\hline Dacnis cayana* & Blue Dacnis & & ES & \\
\hline Coereba flaveola & Bananaquit & & SA/SC/RC/EA/ES & \\
\hline Sporophila lineola ${ }^{\sharp \mathbf{P M}}$ & Lined Seedeater & & & \\
\hline Sporophila nigricollis & Yellow-bellied Seedeater & & SA & \\
\hline Sporophila leucoptera ${ }^{\#}$ & White-bellied Seedeater & & & \\
\hline Sporophila bouvreuil ${ }^{\# \mathrm{PM}}$ & Copper Seedeater & & & \\
\hline Sporophila albogularis & White-throated Seedeater & $\mathrm{EC}$ & $\mathrm{SA} / \mathrm{SC} / \mathrm{RC}$ & WA2918631 \\
\hline Saltator similis & Green-winged Saltator & & SA & \\
\hline Thlypopsis sordida & Orange-headed Tanager & & SA/ES & WA2687083 \\
\hline \multicolumn{5}{|l|}{ CARDINALIDAE } \\
\hline Piranga flava* & Hepatic Tanager & & EA/ES & \\
\hline Cyanoloxia brissonii & Ultramarine Grosbeak & & SA/SC/RC & \\
\hline \multicolumn{5}{|l|}{ FRINGILLIDAE } \\
\hline Spinus yarrellii ${ }^{\#}$ & Yellow-faced Siskin & VU & & \\
\hline Euphonia chlorotica & Purple-throated Euphonia & & SA/SC/RC/EA/ES & \\
\hline \multicolumn{5}{|l|}{ ESTRILDIDAE } \\
\hline Estrilda astrild* & Common Waxbill & & SA & \\
\hline \multicolumn{5}{|l|}{ PASSERIDAE } \\
\hline Passer domesticus & House Sparrow & & SA & \\
\hline
\end{tabular}

sites was fairly complete. Based on our point counts, estimated species richness was 158 (Chao 1) and 167 species (Jackknife 1). Thus, observed richness by point counts corresponds to $95.6 \%$ and $90.4 \%$, respectively, of the estimated richness (Fig. 3).

During point counts, we made 18,272 avian contacts. The 10 most detected species during these censuses were Zonotrichia capensis $(n=926)$, Eupsittula cactorum $(n=$ 850), Zenaida auriculata $(n=675)$, Sakesphorus cristatus $(n=626)$, Stigmatura napensis $(n=620)$, Hemitriccus margaritaceiventer $(n=592)$, Columbina picui $(n=559)$, Polioptila plumbea $(n=547)$, Coryphospingus pileatus ( $n=545)$, and Thamnophilus capistratus $(n=539)$. On the other hand, 26 species were only recorded once (singletons) or twice (doubletons).

During the dry season we detected 117 species and 4,521 individuals. During the wet season (which we sampled twice) we detected 146 species and had a mean abundance of $6,875.2$ individuals $(n=13,751)$. The five most abundant species during the dry season were Eupsittula cactorum ( $n=283$ individuals detected), Chlorostilbon lucidus $(n=276), H$. margaritaceiventer $(n=$ 239), P. plumbea $(n=212)$, and Formicivora melanogaster $(n=174)$. Whereas, during the wet season the five most abundant species were $Z$. capensis $(n=394), Z$. auriculata $(n=337)$, E. cactorum $(n=283), S$. cristatus $(n=243)$ and $S$. napensis $(n=223)$.

From our inventory (systematic and opportunistic) most of the species detected are considered residents. Nineteen species recorded at the CNP are considered migratory or partially migratory (Table 2). For example, Elaenia chilensis is an austral migrant, Tyrannus savana and Turdus amaurochalinus are considered partial austral migrants. We observed a single individual of $T$. savana flying over a disturbed open area in the CNP on March 2017. Elaenia chilensis and T. amaurochalinus were commonly recorded only during the rainy season. Similarly, two species of migratory cuckoos (Coccyzus melacoryphus and Micrococcyx cinereus) were only recorded during the rainy season. Whereas C. melacoryphus was 


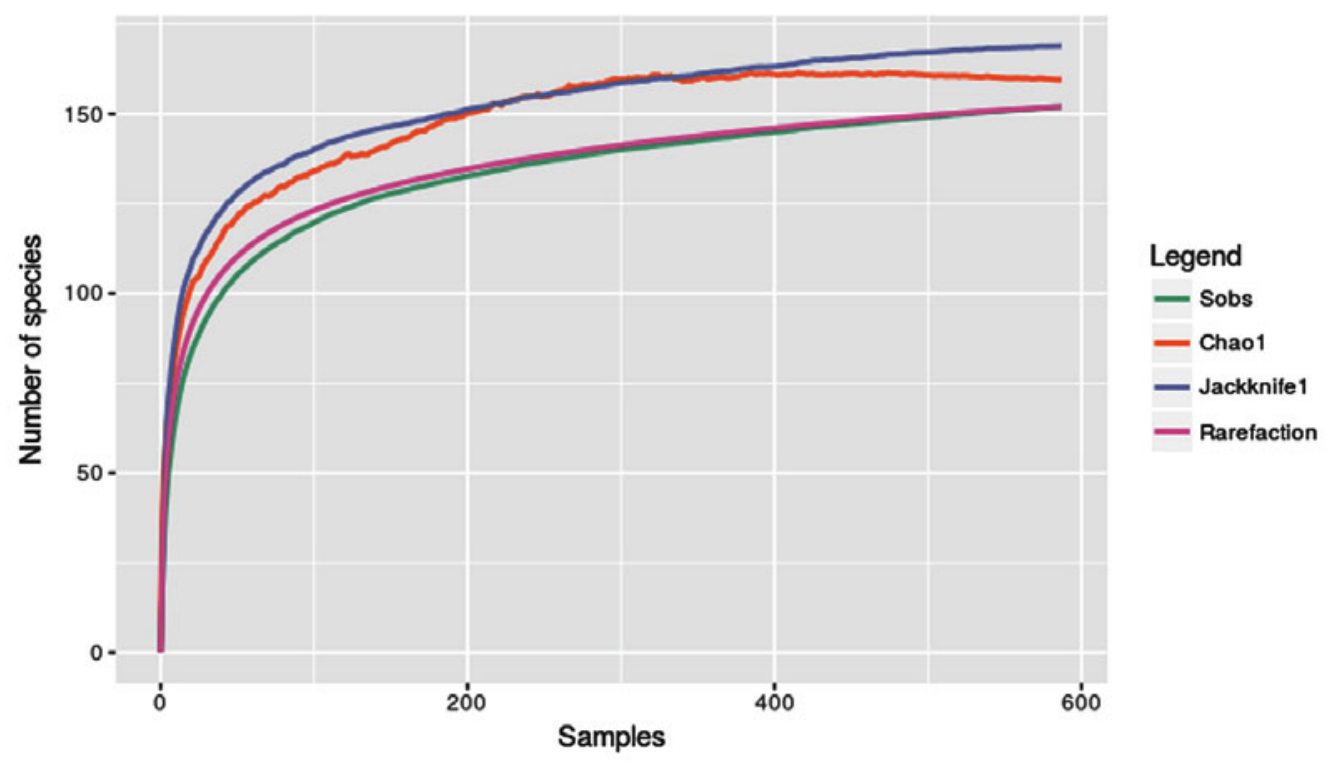

Figure 3. Observed richness (green line), rarefaction (pink line) and richness estimators Chao 1 (orange line) and Jackknife 1 (blue line) curves for the bird assemblage recorded in the Catimbau National Park, Pernambuco, Brazil.

relatively common, $M$. cinereus was recorded once on June 2017, during an opportunistic observation in a disturbed area. Some partial migrant species (Myiophobus fasciatus, Casiornis fuscus, Myiodinastes maculatus, Empidonomus varius, Hydropsalis parvula, Vireo chivi, Pachyramphus polychopterus, Elaenia spectabilis, Myiarchus swainsoni and Progne chalybea) were regularly detected during the rainy season in the park. Finally, species like Bubulcus ibis, Xenopsaris albinucha, Columbina minuta, Columbina talpacoti, Patagioenas picazuro, Z. auriculata, Chrysomus ruficapillus, Agelaioides fringillarius, Molothrus bonariensis, Sicalis luteola, Volatinia jacarina, and aquatic species such as Dendrocygna viduata, Himantopus mexicanus, as well as species from the families Podicipedidae, Ardeidae and Rallidae (Table 2) were recorded exclusively during the rainy season and are likely to carry out seasonal displacements within the Caatinga.

During our surveys most species were detected in more than one habitat. Most of the species recorded during our studies were detected in shrubby-arboreal Caatinga (162 species), followed by shrubby Caatinga with Cerrado elements $(n=85$ spp.), shrubby Caatinga with elements of rocky fields ( $n=59$ spp.), evergreen arboreal vegetation ( $n=56$ spp.), evergreen shrubby Caatinga $(n=89)$, and 12 species related to aquatic environments.

A total of 28 taxa detected in our surveys are considered range-restricted. Nine species are endemic to the Caatinga, whereas 19 taxa are restricted to the Brazilian northeast (Table 2). Picumnus fulvescens a northeastern endemic and Hylopezus ochroleucus a Caatinga endemic are considered "Near Threatened", with decreasing trends in their populations (IUCN 2019). None of the species is considered threatened by extinction according to the Brazilian MMA (2018b). Three exotic species were recorded (Columba livia, Estrilda astrild and Passer domesticus), mostly in urban and peri-urban areas, but there is no evidence that they represent any threat to autoctonous species.

\section{DISCUSSION}

In this study we presented newly quantitative data on the avian assemblage found at the Catimbau National Park. During our quantitave surveys, restricted to 20 sites, we detected $-70 \%$ (155 species) of the 192 species. In addition, opportunistic observations included another 37 species of birds, mostly waterbirds that do not occur at or near our sampled sites. In fact, according to species richness estimators, our quantitative surveys detected the vast majority of the species present in our sites, showing the importance of conducting systematic surveys. The CNP avian assemblage (192 species) represented $-35 \%$ of bird species registered for the Caatinga Domain (sensu Araújo \& Silva $2017 ; n=548)$ and $35.8 \%$ of the 535 bird species recorded for the state of Pernambuco (Farias $\&$ Pereira 2009).

Despite our systematic surveys, we failed to find 34 bird species previously reported for the CNP by Sousa $e t$ al. (2012). Among the species we failed to record, 11 are linked to aquatic environments, including three species of herons (Tigrisoma lineatum, Nycticorax nycticorax, and Butorides striata), two of ducks (Cairina moschata and Amazonetta brasiliensis), two common inhabitants of ponds and pools (Podilymbus podiceps and Nannopterum brasilianus), two species of raptors likely occurring in low densities (Buteo nitidus and Buteo albonotatus), two species 
of hummingbirds (Anthracothorax nigricollis and Polytmus guainumbi), two species of nightbirds (Antrostomus rufus and Chordeiles acutipennis), four species of birds often linked to more humid forests (Herpsilochmus atricapillus, Sittasomus griseicapillus, Synallaxis hypospodia, and Pheugopedius genibarbis), and three species of seedeaters known to have erratic populations elsewhere in the Caatinga (Sporophila lineola, Sporophila leucoptera, and Sporophila bouvreuil).

More important, however, were the apparentabsences of three species of conservation concern, including three endemic and threatened species (Crypturellus noctivagus zabele, P. jacucaca, and S. yarrellii) previously reported by Sousa et al. (2012). These species are known to be widely hunted by poachers and for the illegal trade, and their absences may indicate local extinctions. The CNP suffers strong pressure from hunting and illegal trade, particulalry for birds. During the study period hunters and local residents were observed trapping birds within the CNP boundaries, an illegal activity outside and even inside the protected area. We found many endemic avian taxa in captivity, including E. cactorum, Paroaria dominicana, Sporophila albogularis and Icterus jamacaii, but also more widespread species, such as Amazona aestiva and Cyanoloxia brissonii, widely appreciated by the illegal trade. We are afraid that if the scenario of hunting activities, illegal logging, overgrazing by goats and cattle, hunting of wild animals (mainly mammals and birds), and bird trapping continue to occur within the park, other species may also become locally extinct. Species whose populations are locally and regionally small are more susceptible to local extinction (e.g., Pereira \& Brito 2005, Pereira \& Azevedo-Jr. 2011, Fernandes-Ferreira $e t$ al. 2012, Las-Casas et al. 2012, Albuquerque et al. 2017).

On the other hand, we recorded 25 species that were not previously recorded at the CNP (Table 2), including two aquatic species (Tachybaptus dominicus and Himantopus mexicanus), three species of raptors (Heterospizias meridionalis, Urubitinga urubitinga, Parabuteo unicinctus); some austral and intratropical migrants (M. cinereus, Serpophaga subcristata, T. savana, and Piranga flava), whose movements are poorly known; a boreal unreported fron the park; migrant (Falco peregrinus); two species of exotic birds (Columba livia and Estrilda astrild), previously unreported from the park; and one endemism that likely went undersampled in the past (Herpsilochmus sellowi). These results suggest that the core avian assemblage of the CNP is likely very well established by now, and that future records will likely result from more nomadic aquatic species, austral and northern migrants, and possibly some widespread species that have not yet been recorded in the park. Species richness and avian composition may also vary according to differences in the methods applied, sampling effort, nocturnal observations (Vizentin-Bugoni et al. 2015), as well as the conservation status of the areas (Sayer et al. 2017, Bovo et al. 2018).

These results also suggest that the Caatinga bird assemblage composition presents some clear interannual variation, particularly for aquatic and low density species (Araújo \& Silva 2017). During the rainy season at the CNP, there was an increment in bird species richness, with the presence of migratory birds, including both longdistance and intratropical migrants (e.g., Ruiz-Esparza $e t$ al. 2011, Las-Casas et al. 2012, Lyra-Neves et al. 2012, Araújo et al. 2017).

Most of the bird species found at the CNP occured in shrubby arboreal Caatinga, which is the main phytophisiognomy found within the park boundaries. On the other hand, many species of birds can be found in more than one habitat (Table 2), a pattern that is common among birds in the Caatinga. Most of the species included in the park's list are not forest dependent, being able to explore different habitats (Araújo \& Silva 2017). However, some species may be considered forest specialists. In the CNP species such as Leptotila rufaxilla and Ortalis araucuan were restricted to more humid habitats such as evegreen forests, a type of vegetation nowadays very uncommon within the park. This type of vegetation was transformed in areas of plantations and pastures (e.g., Pedra do Cachorro) and the remaining tracts of evergreen forests is very fragmented and present different levels of disturbances.

We also noticed that some bird species at the CNP prefer well-conserved and/or forested habitats and rarely occur in disturbed environments (Pereira \& AzevedoJr. 2011, Las-Casas et al. 2012, Lyra-Neves et al. 2012). This was the case of P. superciliaris, Trogon curucui, Piculus chrysochloros, Micrastur ruficollis, H. sellowi, H. ochroleucus, C. trochilirostris, Dendroplex picus and S. ruficapillus. In contrast, other species were only observed in disturbed and open areas, such as Athene cunicularia, Suiriri suiriri bahiae and Xolmis irupero niveus. Species such as Sarcoramphus papa, Geranoaetus melanoleucus, Hydropsalis longirostris, Hirundinea ferruginea and Knipolgeus nigerrimus were associated to the CNP's rocky walls. Some of those rare species were those more dependent on forested habitats and more sensitive to disturbance, preferring isolated sites with very low human interference such as P. superciliaris, Claravis pretiosa, M. ruficollis, C. trochilirostris and S. ruficapillus.

The Caatinga is the largest block of tropical Dry forests found within South America (Silva \& Souza 2018) and is one of the most threatened in the Neotropics, with less than $10 \%$ of its original extent (Banda et al. 2016). In Brazil, habitat conservation is uneven among biomes (Jenkins \& Joppa 2009, Oliveira \& Bernard 2017) and the Caatinga represents the least protected one, with only 
$1.3 \%$ of the total area officially included in protected areas that receive full protection (MMA 2017).

Besides the anthropogenic pressures found within this protected area, such as bird hunting, trapping and cattle grazing, our results demonstrate that the CNP still harbors a valuable Caatinga avian diversity with the presence of range-restricted, endemic, threatened, and migratory species, highlighting its importance for bird conservation. But we emphasize the need of effective management inside and outside the park's boundaries, since pressures inside the reserve may usually reflect those occurring around (Laurance et al. 2012). Despite being fragmented, patches of Caatinga remain well-connected, which may facilitate recolonizations and community regeneration (Antongiovanni et al. 2018). Thus, the maintenance, management and expansion of protected area networks continue to be one of the most important tools for biodiversity conservation (Las-Casas et al. 2012, Oliveira \& Bernard 2017, Antongiovanni et al. 2018).

The exceptional natural features of the park, allied to a rich avifauna could provide an economic opportunity through the development of birdwatching, offering new job opportunities. We emphasize that actions such as environmental education and ecological restoration projects, allied to inspection are urgent for the maintenance of the biodiversity and ecosystem services at the CNP.

\section{ACKNOWLEDGEMENTS}

We are grateful to CNPq and FACEPE for providing financial support to F.M.G.L.C. during her post-doctoral project (DCR-0018-2 05/15). We are grateful to Marcelo Tabarelli and Inara Leal, who invited us to lead the avian studies as part of the Long-termed Ecological Monitoring Programme in the park (PELD Catimbau). We are also grateful to Bruna Mirely, Lucas Bueno Jonatham Ramos research at the guide Genivaldo with field assistance, and IBAMA/SISBIO for the licence to conduct this research at the CNP (No. 52032).

\section{REFERENCES}

Albuquerque U.P., Araújo E.L., de Castro C.C. \& Alves R.R.N. 2017. People and natural resources in the Caatinga, p. 303-333. In: Silva J.M.C., Tabarelli M. \& Leal I.R. (eds.). Caatinga: the largest Tropical Dry Forest region in South America. New York: Springer.

Andrade-Lima D. 1981. The Caatinga Dominium. Revista Brasileira de Botânica 4: 149-153.

Antongiovanni M., Venticinque E.M. \& Fonseca C.R. 2018. Fragmentation patterns of the Caatinga drylands. Landscape Ecology 33: 1353-1367.

Araújo H.F.P. \& Silva J.M.C. 2017. The avifauna of the Caatinga: biogeography, ecology, and conservation, p. 181-210. In: Silva
J.M.C., Tabarelli M. \& Leal I.R. (eds.). Caatinga: the largest Tropical Dry Forest region in South America. New York: Springer.

Araújo H.F.P., Vieira-Filho A., Barbosa M.R.V., Diniz-Filho J.A. \& Silva J.M.C. 2017. Passerine phenology in the largest Tropical Dry Forest of South America: effects of climate and resource availability. Emu 117: 78-91.

Arnan X., Leal I.R., Tabarelli M., Andrade J.F., Barros M.F., Câmara T., Jamelli D., Knoechelmann C.M., Menezes T.G.C., Menezes A.G.S., Oliveira F.M.P., de Paula A.S., Pereira S.C., Rito K.F., Sfair J.C., Siqueira F.F.S., Souza D.G., Specht M.J., Vieira L.A., Arcoverde G.B. \& Andersen A.N. 2018. A framework for deriving measures of chronic anthropogenic disturbance: surrogate, direct, single and multi-metric indices in Brazilian Caatinga. Ecological Indicators 94: 274-282.

Banda K., Delgado-Salinas A., Dexter K.G., Linares-Palomino R., Olivera-Filho A., Prado D., Pullan M., Quintana C., Riina R., Rodríguez G.M., Weintritt J., Acevedo-Rodríguez P., Adarve J., Álvarez E., Aranguren A., Arteaga J.C., Aymard G., Castaño A., Ceballos-Mago N., Cogollo A., Cuadros H., Delgado F., Devia W., Dueñas H., Fajardo L., Fernández A., Fernández M.A., Franklin J., Freid E.H., Galetti L.A., Gonto R., González R., Graveson R., Helmer E.H., Idárraga A., López R., Marcano-Vega H., Martínez O.G., Maturo H.M., McDonald M., McLaren K., Melo O., Mijares F., Mogni V., Molina D., Moreno N.P., Nassar J.M., Neves D.M., Oakley L.J., Oatham M., Olvera-Luna A.R., Pezzini F.F., Dominguez O.J.R., Ríos M.E., Rivera O., Rodríguez N., Rojas A., Särkinen T., Sánchez R., Smith M., Vargas C., Villanueva B. \& Pennington R.T. 2016. Plant diversity patterns in Neotropical Dry Forests and their conservation implications. Science 353: 1383-1387.

Bibby C.J., Burgess N.D., Hill D.A. \& Mustoe S.H. 2000. Bird census techniques. London: Academic Press.

Bovo A.A.A., Ferraz K.M.P.M.B., Magioli M., Alexandrino E.R., Hasui E., Ribeiro M.C. \& Tobias J.A. 2018. Habitat fragmentation narrows the distribution of avian functional traits associated with seed dispersal in Tropical Forest. Perspectives in Ecology and Conservation 16: 90-96.

Colwell R.K. 2011. EstimateS: statistical estimation of species richness and shared species from samples. Version 9. User's guide and application. http://purl.oclc.org/estimates (Access on 29 July 2019).

Cruz R., Ramos S.M.S., Fonseca J.C., Motta C.M.S. \& Moreira K.A. 2017. Anthropization effects on the filamentous fungal community of the Brazilian Catimbau National Park. Revista Brasileira de Ciência do Solo 41: e0160373.

Devenish C., Díaz Fernández D.F., Clay R.P., Davidson I. \& YépezZabala I. 2009. Important Bird Areas Americas: priority sites for biodiversity conservation. Quito: BirdLife International.

Egler W.A. 1951. Contribuição ao estudo da Caatinga pernambucana. Revista Brasileira de Geografia 13: 577-590.

Farias G.B. 2009. Aves do Parque Nacional do Catimbau, Buíque, Pernambuco, Brasil. Atualidades Ornitológicas 147: 36-39.

Farias G.B. \& Pereira G.A. 2009. Aves de Pernambuco: o estado atual do conhecimento ornitológico. Biotemas 22: 1-10.

Fernandes-Ferreira H., Mendonça S.V., Albano C., Ferreira F.S. \& Alves R.R.N. 2012. Hunting, use and conservation of birds in northeast Brazil. Biodiversity and Conservation 21: 221-244.

Gotelli N.J. \& Colwell R.K. 2010. Estimating species richness, p: 39-54 In: Magurran A.E. \& McGill B.J. (eds.). Biological diversity: frontiers in measurement and assessment. Oxford: Oxford University Press.

IUCN 2019. The IUCN red list of threatened species. Version 2019.1. http://www.iucnredlist.org (Access on 11 March 2019).

Jenkins C.N. \& Joppa L. 2009. Expansion of the global terrestrial protected area system. Biological Conservation 142: 2166-2174.

Las-Casas F.M.G., Azevedo-Jr. S.M., Dias M.M. \& Bianchi C.A. 2012. Community structure and bird species composition in a 
Caatinga of Pernambuco, Brazil. Revista Brasileira de Ornitologia 20: 302-311.

Laurance W.F. and 215 coauthors. 2012. Averting biodiversity collapse in Tropical Forest protected areas. Nature 489: 290-294.

Leal I.R., Tabarelli M. \& Silva J.M.C. 2003. Ecologia e conservação da Caatinga: uma introdução ao desafio, p: 13-16. In: Leal I.R., Tabarelli M. \& Silva J.M.C. (eds.). Ecologia e conservação da Caatinga. Recife: Editora Universitária.

Lyra-Neves R.M., Azevedo-Jr. S.M., Telino-Jr. W.R., Larrazábal M.E.L. 2012. The birds of the Talhado do São Francisco Natural Monument in the Semi-Arid Brazilian northeast. Revista Brasileira de Ornitologia 20: 268-289.

Magurran A.E. 2004. Measuring biological diversity. Oxford: Blackwell Publishing.

Menezes R.S.C., Sampaio E.V.S.B., Giongo V. \& Pérez-Marin A.M. 2012. Biogeochemical cycling in terrestrial ecosystems of the Caatinga Biome. Brazilian Journal of Biology 72: 643-653.

MMA [Ministério do Meio Ambiente]. 2017. Cadastro Nacional de Unidades de Conservação. http://www.mma.gov.br/images/ arquivo/80112/CNUC_FEV17\%20-\%20C_Bio.pdf (Access on 21 February 2018).

MMA [Ministério do Meio Ambiente]. 2018a. Unidades de Conservaçẫo: Caatinga. http://www.mma.gov.br/biomas/ Caatinga. (Access on 22 February 2018).

MMA [Ministério do Meio Ambiente]. 2018b. Lista Nacional Oficial de espécies da fauna ameaçadas de extinção, Portaria MMA No. 444 de 17 de dezembro de 2014. Diário Oficial da União 245: $121-126$.

Oliveira A.P.C. \& Bernard E. 2017. The financial needs $v s$. the realities of in situ conservation: an analysis of federal funding for protected areas in Brazil's Caatinga. Biotropica 49: 745-752.

Pacheco J.F. 2004. As aves da Caatinga: uma análise histórica do conhecimento, p: 189-250. In: Silva J.M.C., Tabarelli M., Fonseca M.T. \& Lins L.V. (eds.). Biodiversidade da Caatinga: áreas e açôes prioritárias para a conservação. Brasília: Ministério do Meio Ambiente.

Pennington R.T., Prado D.E. \& Pendry C.A. 2000. Neotropical Seasonally Dry Forests and Quaternary vegetation changes. Journal of Biogeography 27: 261-273.

Pennington R.T., Lavin M. \& Oliveira-Filho A. 2009. Woody plant diversity, evolution, and ecology in the tropics: perspectives from Seasonally Dry Tropical Forests. Annual Review of Ecology, Evolution, and Systematics 40: 437-457.

Pereira G.A. \& Azevedo-Jr. S.M. 2011. Estudo comparativo entre as comunidades de aves de dois fragmentos florestais de Caatinga em Pernambuco, Brasil. Revista Brasileira de Ornitologia 19: 22-31.

Pereira G.A. \& Brito M.T. 2005. Diversidade de aves silvestres brasileiras comercializadas nas feiras livres da regiáo metropolitana do Recife, Pernambuco. Atualidades Ornitológicas 126: 14-19.

Piacentini V.Q., Aleixo A., Agne C.E., Maurício G.N., Pacheco J.F., Bravo G.A., Brito G. R.R., Naka L.N., Olmos F., Posso S. Silveira L.F., Betini G.S., Carrano E., Franz I., Lees A.C., Lima L.M., Pioli D., Schunk F., Amaral F.R., Bencke G.A., Cohn-Haft M., Figueiredo L.F.A., Straube F.C. \& Cesari E. 2015. Annotated checklist of the birds of Brazil by the Brazilian Ornithological Records Committee. Revista Brasileira de Ornitologia 23: 91-298.

Ralph C.J., Geupel G.R., Pyle P., Martin T.E., Sante D.F. \& Milá B. 1996. Manual de métodos de campo para el monitoreo de aves terrestres. General Technical Report PSW GTR-159. Albany: Pacific Southwest Research Station.

Rito K.F., Arroyo-Rodríguez V., Queiroz R.T., Leal I.R. \& Tabarelli M. 2017. Precipitation mediates the effect of human disturbance on the Brazilian Caatinga vegetation. Journal of Ecology 105: 828838.

Rodal M.J.N., Andrade K.V.A., Sales M.F. \& Gomes A.P.S. 1998. Fitossociologia do componente lenhoso de um refúgio vegetacional no município de Buíque, Pernambuco. Revista Brasileira de Biologia 58: 517-526.

Ruiz-Esparza J., Rocha P.A., Ruiz-Esparza D.P.B., Ribeiro A.S. \& Ferrari S.F. 2011. Migratory birds in the semi-arid Caatinga scrublands of northeastern Brazil: diversity and seasonal patterns. Ornitología Neotropical 22: 15-24.

Sarmiento G. 1975. The dry plant formations of South America and their floristic connections. Journal of Biogeography 2: 233-251.

Sayer C.A., Bullock J.M. \& Martin P.A. 2017. Dynamics of avian species and functional diversity in secondary Tropical Forests. Biological Conservation 211: 1-9.

Silva J.M.C. \& Barbosa L.C.F. 2017. Impact of human activities on the Caatinga, p. 359-368. In: Silva J.M.C., Leal I.R. \& Tabarelli M. (eds.). Caatinga: the largest Tropical Dry Forest region in South America. Cham: Springer.

Silva A.C. \& Souza A.F. 2018. Aridity drives plant biogeographical sub regions in the Caatinga, the largest Tropical Dry Forest and woodland block in South America. PLoS One 13: e0196130.

SNE [Sociedade Nordestina de Ecologia]. 2002. Projeto técnico para a criação do Parque Nacional do Catimbau/PE. Recife: Secretaria de Ciência, Tecnologia e Meio Ambiente de Pernambuco-SECTMA.

SNUC [Sistema Nacional de Unidades de Conservação da Natureza]. 2002. http://www.mma.gov.br/estruturas/sbs_dap/_arquivos/ snuc_lei_decreto.pdf (Access on 15 September 2017.

Somenzari M., Amaral P.P., Cueto V.R., Guaraldo A.C., Jahn A.E., Lima D.M., Lima P.C., Lugarini C., Machado C.G., Martinez J., Nascimento J.L.X., Pacheco J.F., Paludo D., Prestes N.P., Serafini P.P., Silveira L.F., Sousa A.E.B.A., Sousa N.A., Souza M.A., Telino-Jr. W.R. \& Whitney B.M. 2018. An overview of migratory birds in Brazil. Papéis Avulsos de Zoologia 58: e20185803.

Sousa A.E.B.A., Lima D.M. \& Lyra-Neves R.M. 2012. Avifauna of the Catimbau National Park in the Brazilian state of Pernambuco, Brazil: species richness and spatio-temporal variation. Revista Brasileira de Ornitologia 20: 230-245.

Sutherland W.J., Newton I. \& Green R.E. 2004. Bird ecology and conservation: a handbook of techniques. Oxford: Oxford University Press.

Vizentin-Bugoni J., Jacobs F.P., Coimbra M.A.A., Dias R.A. 2015. Birds of the Reserva Biológica do Mato Grande and surroundings, Rio Grande do Sul, Brazil. Check List 11: 1641.

Associate Editor: Caio G. Machado. 